\title{
Network Security Threat Situation Evaluation Based on Fusion Decision and Spread Analysis
}

\author{
Xiangdong Cai \\ Harbin University of Science and Technology, Harbin, China
}

\begin{abstract}
Most situation evaluation methods suffer from the false positives and false negatives of detection systems seriously, without considering authorization and dependence relationships, unable to reflect indirect threats, and whose assessment results guide dynamic defense poorly. Upon these problems, an evaluation method whose core consists of multi-source fusion decision, threat spread analysis and attack intention guess is presented. First, the decision-level fusion of multi-source detection logs and attack alerts is introduced to improve detection rate or reduce false alarm rate. Afterwards, the direct threats imposed by attacks, the indirect threats caused by spreading along dependence relationships, and the nonlinear overlapping effects under multiple concurrent attacks are evaluated. Finally, covering and clustering method is utilized to guess attack intentions. Experiments show that the method proposed can not only weaken the impact imposed on assessment result by false positive or false negative effectively, reveal security situation more deeply and accurately, but also guide dynamic defense preferably.
\end{abstract}

Keywords: network security, threat situation, situation evaluation, fusion decision, spread analysis, attack intention

\section{Introduction}

Along with the network increasingly large, complex and heterogeneous, security threats are becoming diversified, facing with a large number of different formats, different forms of logs and alerts, the traditional assessment methods already overburdened, thus deriving threat situation assessment. Filtering, integration and abstraction of multi-source information from regulatory facilities, making managers to have a general understanding of the security situation, making a quick response to the complex security threat [1-2].

There are some problems including: (1) Confined to the traditional weighting scheme, requiring the sum of the weights as 1 , this resulted in the presence of constraints elimination relationship between each other, artificial set is very difficult when a slightly larger scale appears [3]. (2) Macro discussed more, fewer microscopic analysis, it is difficult to reflect the influence of topological relations, causing the conclusion lose precision. (3) We are lack of a feasible scheme of multi-source information fusion [4]. (4) Separating application services on the lower dependence of basic services, ignoring the dependence of data service [5]. (5) the most concern is the attacker's intention, the countermeasures to be taken after managers get the evaluation conclusion; most of the methods avoid the problem [6-7].

To solve the above problems, this paper presents a decision based on multi-source fusion, to attack intention guess for extending dynamic assessment method, on the basis of resource value, security vulnerabilities, intrusion alarm, and dependence of threat assessment, could eliminate or weaken the area lack of preceding paragraphs. 


\section{Fusion Decision}

Combining to use of $m$ intrusion detection systems, for a network access $x$, each system independently judges $x$ is a normal visit or some sort of attack. After accumulating a certain number of statistical samples, weakening influence the decision-making level fusion that using false positives or false negatives for assessment [8].

\subsection{Training Model}

Taking $d o$ as the input, $\sigma$ as the expected output, constituting the training samples. Taking the scale count value $\left|X_{\lambda}\right|$ and $\left|X_{\lambda}^{(\sigma)}\right|$ as the parameters to be adjusted. Making mappings $\boldsymbol{d}^{\circ}$ to $\lambda$ firstly, then putting $\left|X_{\lambda}\right|$ and $\left|X_{\lambda}^{(\sigma)}\right|$ plus 1 according to $\lambda$ and $\sigma$, do not have to save the samples. Using $x_{5}$ as the example, the count value $\left|X_{(1,2)}\right|$ and $\left|X_{(1,2)}^{(2)}\right|$ should be cumulated when learning.

\subsection{Decision Method}

When $x$ corresponding to mode $\lambda$, auditing concluded that the conditional probability of $\sigma$ is $P(\operatorname{Audit}(x)=\sigma \mid M a p(A \operatorname{lert}(x))=\lambda)$, abbreviated as $P(x: \lambda \rightarrow \sigma)$, according to formula (1), calling for the conclusion of support for $\sigma$.

$$
P(x: \lambda \rightarrow \sigma)=\left|X_{\lambda}^{(\sigma)}\right| /\left|X_{\lambda}\right|
$$

In view of the similarity detection technique, there is a certain correlation among the detection results of various IDS, it is not only difficult to identify, but also difficult to describe, if the probability is derived under the assumption of independence, it will have a significant deviation, so we adopt formula (1).

We know that $\eta$ represents support threshold, the constraint is $0 \leq \eta<1$. Calculating $\Phi$ according to formula (1), and we choose the maximum of support threshold $\alpha_{i}$ as joint decision-making conclusions, if $\Phi=\varnothing$, it is normal.

\section{Evaluation algorithm}

According to the harm that caused to evaluate the threat situation, context of the assessment along attack: the attacker steal authorization through security vulnerabilities or fake login, illegal to read and write data, theft of service, leading to data leakage or damage, service efficiency reduction or suspension, infracting of the confidentiality, integrity, availability of resources, along the dependence of diffusion to a wider scope, causing collateral damage [9].

Algorithm 1 gives the pseudo code description. The second and 15th rows enumerate each active attack circularly, judging action point. The third to ninth rows describe the mapping from stealing permission to infringement of safety according to direct damage. The 10th to 13th rows execute diffusion analysis, describing the indirect transfer induced infringement dependence. The 14th row executes threat index overlay. The 16 th row seeks integrated situation.

Algorithm 1: assess the threat situation

Input: resources, weaknesses, attack, topological relation, and so on.

Output: threat value $(z)$

01. $(\forall(r, c) \in R \times C)(J[r, c] \leftarrow 0)$ 
02. FOR EACH $a \rightarrow s . v$ DO

03. $\Psi \leftarrow \operatorname{Affect}(s . v), \quad \Gamma \leftarrow \operatorname{Expose}(s . v)$

04. FOR EACH $(o, r) \in \Gamma$ DO

05. IF $o=(\mathbb{P}$ AND $r \in D$ THEN $o=(\bullet$

$\psi \leftarrow \psi \mathrm{U}\{(r, \odot)\}$ ENDIF

06. IF $o=®$ AND $r \in D$ THEN

$\psi \leftarrow \psi \mathrm{U}\{(r$, (i) $)\}$ ENDIF

07. IF $o=\odot$ AND $r \in D$ THEN

$\psi \leftarrow \psi \mathrm{U}\{(r$, (a) $)\}$ ENDIF

08. IF $o=\otimes$ AND $r \in S$ THEN

$\psi \leftarrow \Psi \mathrm{U}\{(r, \odot)\}$ ENDIF

09. ENDFOR

10. FOR EACH Unexpanded $(r, c) \in \Psi$

DO

11. IF $r \in D$ AND $c \in\{$ (1), (a) $\}$ THEN

$\Psi \leftarrow \Psi \mathrm{U}(\varphi(\odot, r) \times\{c\})$ ENDIF

12. IF $r \in S$ AND $c \in\{(i)$, (a) $\}$ THEN

$\psi \leftarrow \Psi \mathrm{U}(\varphi(\otimes, r) \times\{c\})$ ENDIF

13. ENDFOR

14. $(\forall(r, c) \in \Psi)(J[r, c] \leftarrow$

$$
1-(1-J[r, c]) \times(1-P(a) \times I[s . v]))
$$

15. ENDFOR

16. $z \leftarrow \sum_{(r, c) \in R \times C}(J[r, c] \times L[r, c])$

\section{Attacking Intent}

According to the intermediate results of evaluation algorithm, we could speculate attack intention further, as an extension of this situation assessment, providing guidance for dynamic defense; it will become a link between each other.

\subsection{Covering Method}

Covering method based on attack count. Using $\vartheta[r, c]$ represents the number of attacks of $(r, c)$, using $\Psi(a)$ represents the set of $\Psi$. Initializing $(\forall(r, c) \in R \times C)(\vartheta[r, c]=0) \quad$ firstly, then counting through $(\forall a \in A)(\forall(r, c) \in \Psi(a))(\vartheta[r, c] \leftarrow \vartheta[r, c]+1)$, and sorting according to $\vartheta[r, c]$.

Taking the count value of maximum safety items as the primary target, times greater regarded as a secondary objective, and so on [10].

\subsection{Clustering Method}

Clustering method based on kernel similarity. Using $K(A)$ represents pit that corresponding to $A$, delimiting it according to formula (2). For example, $K\left(\left\{a_{i}\right\}\right)=\Psi\left(a_{i}\right), K\left(\left\{a_{k}, a_{l}\right\}\right)=\Psi\left(a_{k}\right)$ I $\Psi\left(a_{l}\right)$.

$$
K(A)=\mathrm{I}_{a \in A} \Psi(a)
$$


We could obtain formula (3) according to formula (2), so the intersection of two nuclear is nuclear. For example, we could use $K\left(\left\{a_{k}, a_{l}\right\}\right)=K\left(\left\{a_{k}\right\}\right)$ I $K\left(\left\{a_{l}\right\}\right)$.

$$
K\left(A_{i} \mathrm{U} A_{k}\right)=K\left(A_{i}\right) \text { I } K\left(A_{k}\right)
$$

The similarity between $K\left(A_{i}\right)$ and $K\left(A_{k}\right)$ is calculated according to formula (4). The constraint is $0 \leq \operatorname{sim}\left(K\left(A_{i}\right), K\left(A_{k}\right)\right) \leq 1$.

$$
\operatorname{sim}\left(K\left(A_{i}\right), K\left(A_{k}\right)\right)=\frac{\left|K\left(A_{i}\right) \mathrm{I} K\left(A_{k}\right)\right|}{\left|K\left(A_{i}\right) \mathrm{U} K\left(A_{k}\right)\right|}
$$

We set $M=\{K(\{a\}) \mid a \in A\}$ as initialization. If $K\left(A_{i}\right)$ and $K\left(A_{i}\right)$ is the most similar, then put $A_{i} \mathrm{U} A_{k}$ as the new set $A_{l}$, updating $M$ according to $M \leftarrow\left(M-\left\{K\left(A_{i}\right), K\left(A_{k}\right)\right\}\right) \mathrm{U}\left\{K\left(A_{l}\right)\right\}$, this process is repeated until there is only one element $K\left(A_{j}\right)$ in $M$.

The polymerization locus constitutes a hierarchical clustering of forest, each element as the root in $M$, the composition of a joint attack from $a$. Traced from the root to the leaf for each tree, we know that all levels of target.

\section{Experimental Analysis and Comparison}

\subsection{Multi-source Integration Decisions}

In the total number of visits, normal and attack accounted for $80 \%$ and $20 \%$, respectively, collecting 120 training samples evenly, and could better reflect the traffic characteristics, using 400 evaluation samples.

From table 1, with the help of the decision level fusion, the two systems can make up the disadvantages of each other, inhibiting each other's false alarm. With $\eta$ increases, the detection rate and the false alarm rate decreased obviously, they restrict each other. Zero ratios only show that it did not occur in the experiment or beyond expression accuracy range in the Table.

Table 1. Decision Fusion Effect

\begin{tabular}{cccc}
\hline$\eta$ & Detection rate $(\%)$ & Check loss rate $(\%)$ & False alarm rate $(\%)$ \\
0.00 & 94.64 & 0.00 & 5.37 \\
0.50 & 64.29 & 0.00 & 1.65 \\
0.80 & 42.86 & 0.00 & 0.00 \\
\hline
\end{tabular}

If combined using $m$ same IDS, we could not complement advantages, disadvantages of cancellation, at this time $\lambda$ only full 0 and full 1 two modes, reducing to a single system effect.

\subsection{Threat Evaluation}

Setting $\eta=0.5, a_{1}$ and $a_{2}$ use slammer worm and Blaster worm attacks, $a_{3}$ represents HTTP attack packets, $a_{4}$ guesses passwords according to dictionary, $P\left(a_{1}\right) \sim P\left(a_{4}\right)$ are 0.87、0.64、0.87、0.52. 
Figure 1 show concurrent multi-attack against the diffusion and overlay, circled numbers indicate nodes and edges that belong to diffusion map, solid line and dashed lines show direct, indirect infringement.

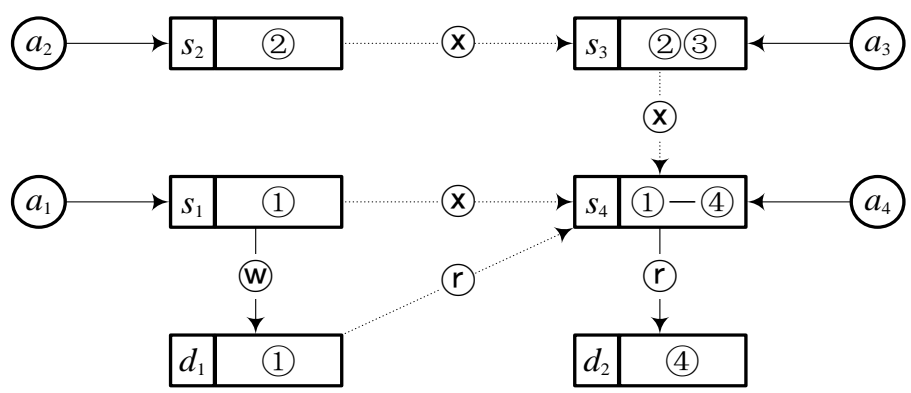

Figure 1. Multiple Attacks Diffusion Analysis

From Figure 2, we could obtain that $z(c)$ is the sub-trend value that relating with $c$, $z=\sum_{c \in C} z(c)$ is the comprehensive trend value, the numbers up the $(t-1, t]$ mark combination attack. For example, [1 3 ] represents $a_{1}$ and $a_{3}$ mark happened, $a_{2}$ and $a_{4}$ didn't happen in $(2,3]$.

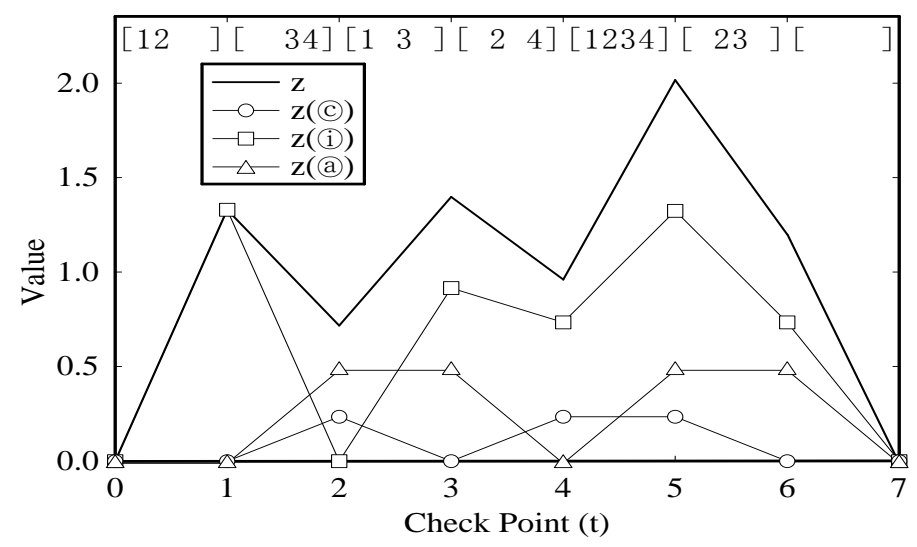

Figure 2. Threat Evaluation Results

If using vector type situation $\boldsymbol{Z}=(z$ (৫) $, z($ (i) $), z$ (@) $)$ replaces the scalar type value $z$, we could reveal the security situation more deeply.

\subsection{Comparison of Related Work}

Advantages of this method are: (1) Using easy to assess and no restriction specific values replace the abstract weights, weakening the dependence on subjective judgments. (2) Most evaluation methods are aiming at "service", this paper take "data" into the scope of assessment, considering authorization from the micro level and dependence, to make a more accurate assessment. (3) This article will alert the vector reflects to several voting patterns, reducing false positives or omissions by decision level fusion, to properly deal with the correlation between the test results, supporting online incremental training. (4) Considering the dependence between services and service data, reflecting the collateral damage caused by infringement of diffusion along dependency 
and risk superposition under multiple concurrent attacks. (5) Speculating on the intent of the attack on the rapid response has certain significance.

Disadvantages of this method are: (1) The automatic identification of poor effect of authorization and dependence, sometimes requiring more artificial setting, after all, the evaluation system is only auxiliary rather than replace the artificial judgment, it is difficult to realize the full automation. (2) The statistical sample number, species and representative to fusion decision making effect larger.

\section{Conclusion}

From the aspect of security elements, the integration of decision-making, evaluation algorithms such as discussed with the intention of attacking threat posture review. In general, with the help of the decision level fusion of multi-source logs and alerts, each detection system can compensate for the lack or inhibition of another false alarm, reducing the combined detection of false positives or omissions, weakening the negative impact on the assessment results. Considering authorization relations, dependencies, topology diffusion, and other factors superimposed danger, to make the assessment result more accurate and credible, and supporting for fine-grained assessment, revealing the security situation more deeply. Aiming at the attack intention speculation to achieve better results, could effectively guide the dynamic defense or quick, enhancing the evaluation results of the practical value.

Further work including: improving on the automatic identification of authorization and dependence; weakening of statistical sample impact on decision fusion effect; improving the accuracy of attack intention to speculate through introducing timing, logic, behavioral correlation analysis.

\section{References}

[1] T. Bass, "Intrusion detection systems and multisensor data fusion: creating cyberspace situational awareness", Communications of the ACM, vol. 43, no. 4, (2002), pp. 99-105.

[2] B. D'Ambrosio, M. Takikawa, D. Upper, et al., "Security situation assessment and response evaluation (SSARE)", In: Proceedings of the DARPA Information Survivability Conference \& Exposition II, Los Alamitos, America: IEEE Computer Society, (2001), pp. 387-394.

[3] X. Chen, Q. Zheng and X. Guan, "Evaluation method of quantitative hierarchical network security threat situation", Journal of software, vol. 17, no. 4, (2006), pp. 885-897.

[4] Y. Liang, H. Wang and J. Lai, "A method of network security situation awareness based on Rough Set Theory", Computer science, vol. 34, no. 8, (2007), pp. 95-97.

[5] H. Xiao, "Analysis and Research on the network security situation assessment and the trend of perception: [PhD thesis]", Shanghai: Shanghai Jiao Tong University School of Electronic Engineering, (2007), pp. 8998.

[6] Y. Wei, Y. Lian and D. Feng, "Network security situation assessment model based on information fusion", Research and development of computer, vol. 46, no. 3, (2009), pp. 353-362.

[7] Y. Wei and Y. Lian, "Situation evaluation model for network security log audit and performance correction algorithm", Chinese Journal of computers, vol. 32, no. 4, (2009), pp. 763-772.

[8] H. R. Shahriari and R. Jalili, "Vulnerability take grant (VTG): an efficient approach to analyze network vulnerabilities", Computers \& Security, vol. 26, no. 5, (2007), pp. 349 360.

[9] Y. Zhang, B. Fang and Y. Chi, "Risk propagation model for the evaluation of network information system", Journal of software, vol. 18, no. 1, (2007), pp. 137-145.

[10] T. Chen, X. Qiao and L. Zheng, "Application of graph theory in threat situation analysis of network security", Journal of Beijing University of Posts and Telecommunications, vol. 32, no. 1, (2009), pp. 113-117. 